\title{
CON: Fomepizole should be used more liberally in paracetamol overdose
}

\author{
James Dear ${ }^{1}$ \\ ${ }^{1}$ Edinburgh University
}

July 10, 2021

\begin{abstract}
Fomepizole is a promising new treatment for preventing liver injury following paracetamol (acetaminophen) overdose. However, we need robust clinical trials to be performed to demonstrate its effect on clinical outcomes that are important to our patients and important to healthcare providers. Until such trials are performed, the toxicology community should learn the lessons from the COVID pandemic - potential novel therapeutic options may be theoretically appealing, but their effectiveness needs to be assessed in robust clinical trials before they are used in clinical practice.
\end{abstract}

Title: The lessons of COVID should guide our use of fomepizole in paracetamol overdose

Running Title: Fomepizole Debate Con

Keywords: Paracetamol, acetaminophen, overdose, fomepizole

Author: James W Dear ${ }^{1}$.

Pharmacology, Therapeutics and Toxicology, Centre for Cardiovascular Science, University of Edinburgh, The Queen's Medical Research Institute, 47 Little France Crescent, Edinburgh, EH16 4TJ

Corresponding author: Professor James W. Dear

University/BHF Centre for Cardiovascular Science

University of Edinburgh

The Queen's Medical Research Institute 47 Little France Crescent Edinburgh EH16 4TJ

james.dear@ed.ac.uk

$(+44) 01312429216$

\section{Abstract}

Fomepizole is a promising new treatment for preventing liver injury following paracetamol (acetaminophen) overdose. However, we need robust clinical trials to be performed to demonstrate its effect on clinical outcomes that are important to our patients and important to healthcare providers. Until such trials are performed, the toxicology community should learn the lessons from the COVID pandemic - potential novel therapeutic options may be theoretically appealing, but their effectiveness needs to be assessed in robust clinical trials before they are used in clinical practice.

The COVID-19 pandemic has dominated healthcare for the last couple of years. The enormous challenge presented by the emergence of this new infectious disease in late 2019 resulted in drug and vaccine development being rapidly performed at 'warp speed' . When COVID was first identified, clinicians searched for licenced 
medicines with mechanisms of action consistent with a potentially effective treatment. Hydroxychloroquine was identified as a potential treatment because it has in vitro activity against SARS-CoV-2[1] and early small observational studies suggested a possible benefit.[2] There were high profile leaders who promoted using hydroxychloroquine to prevent and treat COVID, [3] and doctors prescribed it based on this limited evidence base. Fortunately, from the start of the pandemic, theRecovery platform trial tested multiple treatments in a large randomised clinical trial that measured mortality as the primary endpoint. Recovery conclusively demonstrated hydroxychloroquine had no benefit when 1561 hospitalised patients with COVID were treated with this drug compared to 3155 patients who received standard care.[4] In fact, subsequent a meta-analysis suggests hydroxychloroquine may harm patients.[5] Despite the initial hope that resulted in doctors prescribing hydroxychloroquine, it proved ineffective when tested in a robust large study. Fomepizole may be the toxicology community's hydroxychloroquine unless we learn the lessons of COVID and come together to perform robust randomised trials before prescribing an untested treatment.

Paracetamol (acetaminophen) overdose is common. There is a clear unmet need for new treatments. Currently, the only effective treatment for preventing liver injury after paracetamol overdose is acetylcysteine (n-acetylcysteine, NAC). If treatment is commenced within 8 hours of the overdose, then NAC is near 100\% effective at preventing liver failure. However, its effectiveness drops substantially when treatment is delayed. NAC is near ineffective when treatment is delayed greater than around 20 hours after overdose.[6] For patients who present to hospital late following a significant paracetamol overdose we need effective new treatment strategies to prevent liver injury in this high risk group. Within this space, fomepizole has emerged as a potential new candidate.

Fomepizole is well known to toxicologists as an effective treatment for toxic alcohol poisoning due to its ability to inhibit the enzyme alcohol dehydrogenase.[7] The potential as a treatment for paracetamol overdose is unrelated to this mechanism of action. Fomepizole is a potent inhibitor of the cytochrome P450 enzymes that produce the paracetamol toxic metabolite (NAPQI) that is responsible for liver injury. Furthermore, in mice, fomepizole prevents liver injury after the metabolism phase of paracetamol mainly through the inhibition of c-Jun N-terminal kinase activation. The pre-clinical evidence base for fomepizole is impressive, largely due to the work of Hartmut Jaeschke's group. [8, 9] In humans, well performed clinical studies confirm that fomepizole inhibits the oxidative metabolism of paracetamol.[10] However, there is no trial evidence that fomepizole prevents liver injury in man. These trials need to be performed. Sometimes it is suggested that 'trials cannot be performed in clinical toxicology'. As paracetamol overdose is common this is untrue. In the UK, every 5 minutes someone presents to hospital following a paracetamol overdose - the same frequency as myocardial infarction. It is hard to imagine cardiologists prescribing a medicine because it works in mouse models and defending that position by saying clinical trials cannot be performed in ischaemic heart disease.

There are a number of reasons to insist on robust, randomised trial evidence for the clinical effectiveness of fomepizole before it is used to treat paracetamol overdose in routine clinical practice. Advocates of its use suggest it has a role in large overdoses when there is a high concentration of paracetamol in the circulation. Given fomepizole's ability to inhibit the P450 enzymes this makes theoretical sense. However, P450 enzyme inhibitors have been tested in this indication in the past and failed to demonstrate clinical effectiveness.[11] If P450 enzyme inhibition is the correct target for a new therapy, there may be cheaper alternatives to the expensive option presented by using fomepizole. Finally, and perhaps most importantly, we do not know whether using a higher dose of NAC for larger overdoses will be sufficient alone to prevent liver injury in this group of patients. This approach is likely to be substantially cheaper than using fomepizole. Currently, patients receive a dose of NAC that is based only on their body weight. It is clear from the basic pharmacology, mathematical modelling[12, 13] and observational studies $[14,15][16]$ that patients taking a large overdose may not be receiving enough NAC to prevent liver injury. There are new regimens for administering NAC that produce substantially lower rates of adverse drug reactions compared to the standard 21-hour regimen.[17] These new regimes allow phase 2 clinical trials of high dose NAC treatment in selected patients with a reasonable expectation of not producing dose-limiting toxicity. Before advocating a new expensive treatment, the toxicology community should focus on defining the optimal dose of NAC. 
Fomepizole use in paracetamol overdose is occasionally defended as it is claimed to have an excellent safety profile. This seems to be correct in its licenced indication - treatment of toxic alcohol poisoning. We do not know if it is safe in patients who have overdosed on paracetamol and there are reasons for a degree of caution. A phase 1 trial of fomepizole demonstrated a small increase in alanine transaminase (ALT) activity in 6 of 15 healthy subjects.[18] Although unlikely, when combined with a potentially hepatotoxic dose of paracetamol this small ALT rise could translate into increased cases of significant liver injury. A fomepizole-induced increase in ALT, even if benign, may result in patients having unnecessary prolonged treatment with NAC, as ALT is the key biomarker which informs decisions to stop treatment. We do not have robust data to know the answers to these questions about safety.

Fomepizole has promise as a treatment for paracetamol overdose. But we do not have data from randomised trials about clinically important outcomes such as liver injury, hospital length of stay and liver failure and we do not have an adequate safety dataset. Now is the time for the toxicology community to follow the example of the Recovery Trialand come together to perform large platform trials to evaluate new treatments that have evidence of clinical efficacy from phase 2 trials. Candidate treatments include high dose NAC and fomepizole. This is the hard thing to do, the easier option is to start prescribing treatments without a robust evidence base. The danger is fomepizole becomes like hydroxychloroquine in COVID, advocated as a treatment without robust evidence of benefit for our patients.

Conflict of interest: JWD was the Chief Investigator on the POP Trial of calmangafodipir in paracetamol overdose.

\section{References:}

1. Wang M, Cao R, Zhang L, Yang X, Liu J, Xu M, Shi Z, Hu Z, Zhong W, Xiao G. Remdesivir and chloroquine effectively inhibit the recently emerged novel coronavirus (2019-nCoV) in vitro. Cell Res 2020; 30: $269-71$.

2. Gautret P, Lagier JC, Parola P, Hoang VT, Meddeb L, Mailhe M, Doudier B, Courjon J, Giordanengo V, Vieira VE, Tissot Dupont H, Honore S, Colson P, Chabriere E, La Scola B, Rolain JM, Brouqui P, Raoult D. Hydroxychloroquine and azithromycin as a treatment of COVID-19: results of an open-label non-randomized clinical trial. Int J Antimicrob Agents 2020; 56: 105949.

3. Niburski K, Niburski O. Impact of Trump's Promotion of Unproven COVID-19 Treatments and Subsequent Internet Trends: Observational Study. J Med Internet Res 2020; 22: e20044.

4. Group RC, Horby P, Mafham M, Linsell L, Bell JL, Staplin N, Emberson JR, Wiselka M, Ustianowski A, Elmahi E, Prudon B, Whitehouse T, Felton T, Williams J, Faccenda J, Underwood J, Baillie JK, Chappell LC, Faust SN, Jaki T, Jeffery K, Lim WS, Montgomery A, Rowan K, Tarning J, Watson JA, White NJ, Juszczak E, Haynes R, Landray MJ. Effect of Hydroxychloroquine in Hospitalized Patients with Covid-19. N Engl J Med 2020; 383: 2030-40.

5. Axfors C, Schmitt AM, Janiaud P, Van't Hooft J, Abd-Elsalam S, Abdo EF, Abella BS, Akram J, Amaravadi RK, Angus DC, Arabi YM, Azhar S, Baden LR, Baker AW, Belkhir L, Benfield T, Berrevoets MAH, Chen CP, Chen TC, Cheng SH, Cheng CY, Chung WS, Cohen YZ, Cowan LN, Dalgard O, de Almeida EVFF, de Lacerda MVG, de Melo GC, Derde L, Dubee V, Elfakir A, Gordon AC, Hernandez-Cardenas CM, Hills T, Hoepelman AIM, Huang YW, Igau B, Jin R, Jurado-Camacho F, Khan KS, Kremsner PG, Kreuels B, Kuo CY, Le T, Lin YC, Lin WP, Lin TH, Lyngbakken MN, McArthur C, McVerry BJ, Meza-Meneses P, Monteiro WM, Morpeth SC, Mourad A, Mulligan MJ, Murthy S, Naggie S, Narayanasamy S, Nichol A, Novack LA, O'Brien SM, Okeke NL, Perez L, Perez-Padilla R, Perrin L, Remigio-Luna A, Rivera-Martinez NE, Rockhold FW, Rodriguez-Llamazares S, Rolfe R, Rosa R, Rosjo H, Sampaio VS, Seto TB, Shahzad M, Soliman S, Stout JE, Thirion-Romero I, Troxel AB, Tseng TY, Turner NA, Ulrich RJ, Walsh SR, Webb SA, Weehuizen JM, Velinova M, Wong HL, Wrenn R, Zampieri FG, Zhong W, Moher D, Goodman SN, Ioannidis JPA, Hemkens LG. Mortality outcomes with hydroxychloroquine and chloroquine in COVID-19 from an international collaborative meta-analysis of randomized trials. Nat Commun 2021; 12: 2349. 
6. Smilkstein MJ, Knapp GL, Kulig KW, Rumack BH. Efficacy of oral N-acetylcysteine in the treatment of acetaminophen overdose. Analysis of the national multicenter study (1976 to 1985). N Engl J Med 1988; 319: $1557-62$.

7. Brent J. Fomepizole for ethylene glycol and methanol poisoning. N Engl J Med 2009; 360: 2216-23.

8. Akakpo JY, Ramachandran A, Duan L, Schaich MA, Jaeschke MW, Freudenthal BD, Ding WX, Rumack BH, Jaeschke H. Delayed Treatment With 4-Methylpyrazole Protects Against Acetaminophen Hepatotoxicity in Mice by Inhibition of c-Jun n-Terminal Kinase. Toxicol Sci 2019; 170: 57-68.

9. Akakpo JY, Ramachandran A, Kandel SE, Ni HM, Kumer SC, Rumack BH, Jaeschke H. 4-Methylpyrazole protects against acetaminophen hepatotoxicity in mice and in primary human hepatocytes. Hum Exp Toxicol 2018; 37: 1310-22.

10. Kang AM, Padilla-Jones A, Fisher ES, Akakpo JY, Jaeschke H, Rumack BH, Gerkin RD, Curry SC. The Effect of 4-Methylpyrazole on Oxidative Metabolism of Acetaminophen in Human Volunteers. J Med Toxicol 2020; 16: 169-76.

11. Mullins ME, Yeager LH, Freeman WE. Metabolic and mitochondrial treatments for severe paracetamol poisoning: a systematic review. Clin Toxicol (Phila) 2020; 58: 1284-96.

12. Rumack BH, Bateman DN. Acetaminophen and acetylcysteine dose and duration: past, present and future. Clin Toxicol (Phila) 2012; 50: 91-8.

13. Hendrickson RG. What is the most appropriate dose of N-acetylcysteine after massive acetaminophen overdose? Clin Toxicol (Phila) 2019: 1-6.

14. Cairney DG, Beckwith HK, Al-Hourani K, Eddleston M, Bateman DN, Dear JW. Plasma paracetamol concentration at hospital presentation has a dose-dependent relationship with liver injury despite prompt treatment with intravenous acetylcysteine. Clin Toxicol (Phila) 2016; 54: 405-10.

15. Marks DJB, Dargan PI, Archer JRH, Davies CL, Dines AM, Wood DM, Greene SL. Outcomes from massive paracetamol overdose: a retrospective observational study. Br J Clin Pharmacol 2017; 83: 1263-72.

16. Chiew AL, Isbister GK, Kirby KA, Page CB, Chan BSH, Buckley NA. Massive paracetamol overdose: an observational study of the effect of activated charcoal and increased acetylcysteine dose (ATOM-2). Clin Toxicol (Phila) 2017; 55: 1055-65.

17. Bateman DN, Dear JW, Thanacoody HK, Thomas SH, Eddleston M, Sandilands EA, Coyle J, Cooper JG, Rodriguez A, Butcher I, Lewis SC, Vliegenthart AD, Veiraiah A, Webb DJ, Gray A. Reduction of adverse effects from intravenous acetylcysteine treatment for paracetamol poisoning: a randomised controlled trial. Lancet 2014; 383: 697-704.

18. Jacobsen D, Sebastian CS, Barron SK, Carriere EW, McMartin KE. Effects of 4-methylpyrazole, methanol/ethylene glycol antidote, in healthy humans. J Emerg Med 1990; 8: 455-61. 